Alfio Quarteroni · Alberto Valli

\title{
Numerical Approximation of Partial Differential Equations
}

With 59 Figures and 17 Tables

型 Springer 


\section{Table of Contents}

Part I. Basic Concepts and Methods for PDEs' Approximation

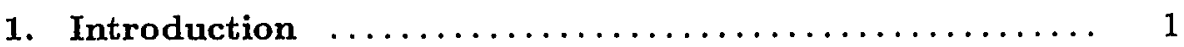

1.1 The Conceptual Path Behind the Approximation ........ 2

1.2 Preliminary Notation and Function Spaces ............ 4

1.3 Some Results About Sobolev Spaces $\ldots \ldots \ldots \ldots \ldots \ldots \ldots .10$

1.4 Comparison Results $\ldots \ldots \ldots \ldots \ldots \ldots \ldots \ldots \ldots \ldots, 13$

2. Numerical Solution of Linear Systems $\ldots \ldots \ldots \ldots \ldots \ldots, 17$

2.1 Direct Methods ........................... 17

2.1 .1 Banded Systems $\ldots \ldots \ldots \ldots \ldots \ldots \ldots \ldots \ldots \ldots, 22$

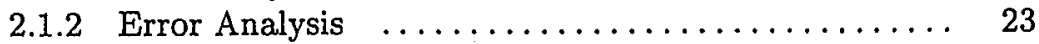

2.2 Generalities on Iterative Methods $\ldots \ldots \ldots \ldots \ldots \ldots \ldots .26$

2.3 Classical Iterative Methods $\ldots \ldots \ldots \ldots \ldots \ldots \ldots \ldots . \ldots \ldots$

2.3.1 Jacobi Method ........................ 29

2.3.2 Gauss-Seidel Method ................... 31

2.3.3 Relaxation Methods (S.O.R. and S.S.O.R.) _..... 32

2.3.4 Chebyshev Acceleration Method .............. 34

2.3.5 The Alternating Direction Iterative Method ...... 37

2.4 Modern Iterative Methods $\ldots \ldots \ldots \ldots \ldots \ldots \ldots \ldots . . \ldots \ldots$

2.4.1 Preconditioned Richardson Method ........... 39

2.4.2 Conjugate Gradient Method $\ldots \ldots \ldots \ldots \ldots \ldots \ldots, 46$

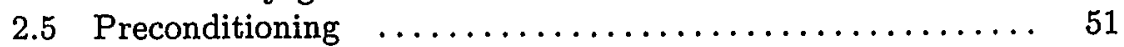

2.6 Conjugate Gradient and Lanczos like Methods for
Non-Symmetric Problems ......................... 57

2.6.1 GCR, Orthomin and Orthodir Iterations ....... 57

2.6.2 Arnoldi and GMRES Iterations . ............. 59

2.6.3 Bi-CG, CGS and Bi-CGSTAB Iterations ......... 62

2.7 The Multi-Grid Method $\ldots \ldots \ldots \ldots \ldots \ldots \ldots \ldots \ldots . \ldots 6$

2.7.1 The Multi-Grid Cycles $\ldots \ldots \ldots \ldots \ldots \ldots \ldots \ldots 6 . \ldots 6$

2.7.2 A Simple Example .................... 67

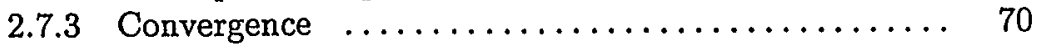

2.8 Complements $\ldots \ldots \ldots \ldots \ldots \ldots \ldots \ldots \ldots \ldots \ldots \ldots \ldots, 71$ 
3. Finite Element Approximation $\ldots \ldots \ldots \ldots \ldots \ldots \ldots \ldots \ldots$

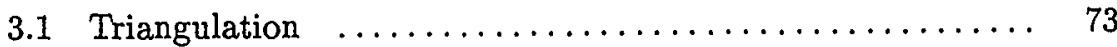

3.2 Piecewise-Polynomial Subspaces $\ldots \ldots \ldots \ldots \ldots \ldots \ldots \ldots \ldots 74$

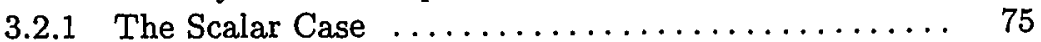

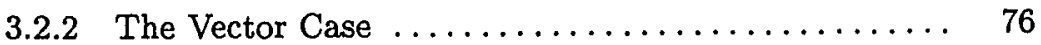

3.3 Degrees of Freedom and Shape Functions ........ . . . 77

3.3.1 The Scalar Case: Triangular Finite Elements ..... 77

3.3.2 The Scalar Case: Parallelepipedal Finite Elements . . 80

3.3.3 The Vector Case . . . . . . . . . . . . . . . 82

3.4 The Interpolation Operator $\ldots \ldots \ldots \ldots \ldots \ldots \ldots \ldots \ldots$

3.4.1 Interpolation Error: the Scalar Case ........... 85

3.4.2 Interpolation Error: the Vector Case $\ldots \ldots \ldots \ldots 9$.

3.5 Projection Operators $\ldots \ldots \ldots \ldots \ldots \ldots \ldots \ldots \ldots \ldots \ldots \ldots . \ldots \ldots$

3.6 Complements $\ldots \ldots \ldots \ldots \ldots \ldots \ldots \ldots \ldots \ldots \ldots \ldots \ldots$

4. Polynomial Approximation $\ldots \ldots \ldots \ldots \ldots \ldots \ldots \ldots \ldots \ldots 10 \ldots$

4.1 Orthogonal Polynomials .................... 101

4.2 Gaussian Quadrature and Interpolation ............ 103

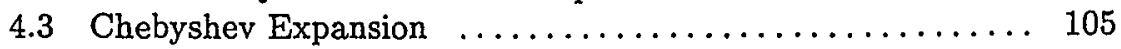

4.3.1 Chebyshev Polynomials $\ldots \ldots \ldots \ldots \ldots \ldots \ldots \ldots \ldots \ldots$

4.3.2 Chebyshev Interpolation $\ldots \ldots \ldots \ldots \ldots \ldots \ldots$

4.3.3 Chebyshev Projections $\ldots \ldots \ldots \ldots \ldots \ldots \ldots \ldots \ldots \ldots$

4.4 Legendre Expansion $\ldots \ldots \ldots \ldots \ldots \ldots \ldots \ldots \ldots \ldots \ldots \ldots$

4.4 .1 Legendre Polynomials $\ldots \ldots \ldots \ldots \ldots \ldots \ldots \ldots \ldots$

4.4 .2 Legendre Interpolation $\ldots \ldots \ldots \ldots \ldots \ldots \ldots \ldots \ldots$

4.4 .3 Legendre Projections $\ldots \ldots \ldots \ldots \ldots \ldots \ldots \ldots \ldots \ldots$

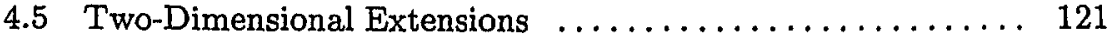

4.5.1 The Chebyshev Case ................. 121

4.5 .2 The Legendre Case $\ldots \ldots \ldots \ldots \ldots \ldots \ldots \ldots \ldots \ldots \ldots$

4.6 Complements $\ldots \ldots \ldots \ldots \ldots \ldots \ldots \ldots \ldots \ldots \ldots \ldots \ldots \ldots$

5. Galerkin, Collocation and Other Methods ........... 129

5.1 An Abstract Reference Boundary Value Problem . . . . . . 129

5.1.1 Some Results of Functional Analysis . . . . . . . 133

5.2 Galerkin Method ....................... 136

5.3 Petrov-Galerkin Method ................... 138

5.4 Collocation Method ....................... 140

5.5 Generalized Galerkin Method ................... 141

5.6 Time-Advancing Methods for Time-Dependent Problems . . 144

5.6.1 Semi-Discrete Approximation $\ldots \ldots \ldots \ldots \ldots \ldots \ldots 148$

5.6.2 Fully-Discrete Approximation $\ldots \ldots \ldots \ldots \ldots \ldots \ldots 148$

5.7 Fractional-Step and Operator-Splitting Methods ....... 151

5.8 Complements $\ldots \ldots \ldots \ldots \ldots \ldots \ldots \ldots \ldots \ldots \ldots \ldots \ldots \ldots$ 
Part II. Approximation of Boundary Value Problems

6. Elliptic Problems: Approximation by Galerkin and Collocation Methods ......................... 159

6.1 Problem Formulation and Mathematical Properties ...... 159

6.1.1 Variational Form of Boundary Value Problems ..... 161

6.1.2 Existence, Uniqueness and A-Priori Estimates ..... 164

6.1.3 Regularity of Solutions .................. 167

6.1.4 On the Degeneracy of the Constants in Stability and Error Estimates $\ldots \ldots \ldots \ldots \ldots \ldots \ldots \ldots, 168$

6.2 Numerical Methods: Construction and Analysis ......... 169

6.2.1 Galerkin Method: Finite Element and Spectral

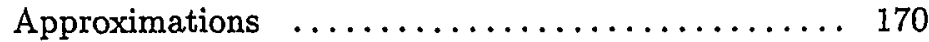

6.2.2 Spectral Collocation Method ............... 179

6.2.3 Generalized Galerkin Method .............. 187

6.3 Algorithmic Aspects $\ldots \ldots \ldots \ldots \ldots \ldots \ldots \ldots \ldots \ldots . . \ldots \ldots$

6.3.1 Algebraic Formulation ................... 190

6.3.2 The Finite Element Case ................. 192

6.3.3 The Spectral Collocation Case $\ldots \ldots \ldots \ldots \ldots \ldots . . \ldots 198$

6.4 Domain Decomposition Methods .................. 204

6.4.1 The Schwarz Method .................. 206

6.4.2 Iteration-by-Subdomain Methods Based on Transmission Conditions at the Interface $\ldots \ldots \ldots .209$

6.4.3 The Steklov-Poincaré Operator .............. 212

6.4.4 The Connection Between Iterations-by-Subdomain Methods and the Schur Complement System ...... 215

7. Elliptic Problems: Approximation by Mixed and

Hybrid Methods ........................... 217

7.1 Alternative Mathematical Formulations ............ 217

7.1.1 The Minimum Complementary Energy Principle .... 218

7.1.2 Saddle-Point Formulations: Mixed and Hybrid Methods .......................... 222

7.2 Approximation by Mixed Methods ............... 230

7.2.1 Setting up and Analysis $\ldots \ldots \ldots \ldots \ldots \ldots \ldots \ldots 230$

7.2.2 An Example: the Raviart-Thomas Finite Elements .. 235

7.3 Some Remarks on the Algorithmic Aspects ........... 241

7.4 The Approximation of More General Constrained

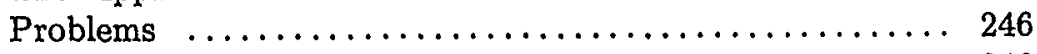

7.4.1 Abstract Formulation .................. 246

7.4.2 Analysis of Stability and Convergence ......... 250

7.4.3 How to Verify the Uniform Compatibility Condition 253

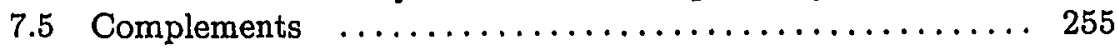


8. Steady Advection-Diffusion Problems $\ldots \ldots \ldots \ldots \ldots \ldots 257$

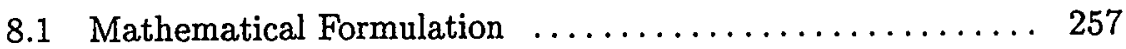

8.2 A One-Dimensional Example ................. 258

8.2.1 Galerkin Approximation and Centered Finite

Differences ........................ 259

8.2.2 Upwind Finite Differences and Numerical Diffusion . 262

8.2.3 Spectral Approximation $\ldots \ldots \ldots \ldots \ldots \ldots \ldots \ldots 263$

8.3 Stabilization Methods ...................... 265

8.3.1 The Artificial Diffusion Method ............ 267

8.3.2 Strongly Consistent Stabilization Methods for Finite Elements ....................... 269

8.3.3 Stabilization by Bubble Functions $\ldots \ldots \ldots \ldots \ldots 273$

8.3.4 Stabilization Methods for Spectral Approximation .. 277

8.4 Analysis of Strongly Consistent Stabilization Methods ..... 280

8.5 Some Numerical Results . . . . . . . . . . . . . . . . . 288

8.6 The Heterogeneous Method $\ldots \ldots \ldots \ldots \ldots \ldots \ldots \ldots .289$

9. The Stokes Problem $\ldots \ldots \ldots \ldots \ldots \ldots \ldots \ldots \ldots \ldots . \ldots \ldots$

9.1 Mathematical Formulation and Analysis ............ 297

9.2 Galerkin Approximation .................... 300

9.2.1 Algebraic Form of the Stokes Problem .......... 303

9.2.2 Compatibility Condition and Spurious Pressure Modes ........................... 304

9.2.3 Divergence-Free Property and Locking Phenomena .. 305

9.3 Finite Element Approximation ................ 306

9.3.1 Discontinuous Pressure Finite Elements ......... 306

9.3.2 Continuous Pressure Finite Elements ........... 310

9.4 Stabilization Procedures $\ldots . \ldots \ldots \ldots \ldots \ldots \ldots \ldots . \ldots . \ldots 11$

9.5 Approximation by Spectral Methods ............. 317

9.5.1 Spectral Galerkin Approximation ............ 319

9.5.2 Spectral Collocation Approximation ........... 323

9.5.3 Spectral Generalized Galerkin Approximation ...... 324

9.6 Solving the Stokes System $\ldots \ldots \ldots \ldots \ldots \ldots \ldots \ldots . \ldots \ldots$

9.6.1 The Pressure-Matrix Method ............... 326

9.6.2 The Uzawa Method ....................... 327

9.6.3 The Arrow-Hurwicz Method ............... 328

9.6.4 Penalty Methods ........................ 329

9.6.5 The Augmented-Lagrangian Method .......... 330

9.6.6 Methods Based on Pressure Solvers ............. 331

9.6.7 A Global Preconditioning Technique .......... 335

9.7 Complements ........................... 337

10. The Steady Navier-Stokes Problem $\ldots \ldots \ldots \ldots \ldots \ldots . . \ldots 339$

10.1 Mathematical Formulation $\ldots \ldots \ldots \ldots \ldots \ldots \ldots \ldots . . \ldots 339$ 
10.1.1 Other Kind of Boundary Conditions ......... 343

10.1.2 An Abstract Formulation . ................ 345

10.2 Finite Dimensional Approximation $\ldots \ldots \ldots \ldots \ldots \ldots \ldots, 346$

10.2.1 An Abstract Approximate Problem ............ 347

10.2.2 Approximation by Mixed Finite Element Methods .. 349

10.2.3 Approximation by Spectral Collocation Methods ... 351

10.3 Numerical Algorithms $\ldots \ldots \ldots \ldots \ldots \ldots \ldots \ldots \ldots \ldots .353$

10.3.1 Newton Methods and the Continuation Method .... 353

10.3.2 An Operator-Splitting Algorithm ............ 358

10.4 Stream Function-Vorticity Formulation of the

Navier-Stokes Equations $\ldots \ldots \ldots \ldots \ldots \ldots \ldots \ldots \ldots \ldots, 359$

10.5 Complements $\ldots \ldots \ldots \ldots \ldots \ldots \ldots \ldots \ldots \ldots \ldots, 361$

Part III. Approximation of Initial-Boundary Value Problems

11. Parabolic Problems $\ldots \ldots \ldots \ldots \ldots \ldots \ldots \ldots \ldots \ldots \ldots \ldots, 363$

11.1 Initial-Boundary Value Problems and Weak Formulation ... 363

11.1.1 Mathematical Analysis of Initial-Boundary Value

Problems .......................... 365

11.2 Semi-Discrete Approximation $\ldots \ldots \ldots \ldots \ldots \ldots \ldots \ldots, 373$

11.2.1 The Finite Element Case $\ldots \ldots \ldots \ldots \ldots \ldots \ldots . \ldots . \ldots 373$

11.2.2 The Case of Spectral Methods .............. 379

11.3 Time-Advancing by Finite Differences $\ldots \ldots \ldots \ldots \ldots \ldots . \ldots 384$

11.3.1 The Finite Element Case $\ldots \ldots \ldots \ldots \ldots \ldots \ldots, 385$

11.3.2 The Case of Spectral Methods .............. 396

11.4 Some Remarks on the Algorithmic Aspects .......... 401

11.5 Complements $\ldots . \ldots \ldots \ldots \ldots \ldots \ldots \ldots \ldots \ldots \ldots . \ldots 404$

12. Unsteady Advection-Diffusion Problems $\ldots \ldots \ldots \ldots \ldots 405$

12.1 Mathematical Formulation $\ldots \ldots \ldots \ldots \ldots \ldots \ldots \ldots .405$

12.2 Time-Advancing by Finite Differences $\ldots \ldots \ldots \ldots \ldots \ldots, 408$

12.2.1 A Sharp Stability Result for the $\theta$-scheme $\ldots \ldots \ldots .408$

12.2.2 A Semi-Implicit Scheme ................ 411

12.3 The Discontinuous Galerkin Method for Stabilized

Problems .............................. 415

12.4 Operator-Splitting Methods $\ldots \ldots \ldots \ldots \ldots \ldots \ldots \ldots, 418$

12.5 A Characteristic Galerkin Method .............. 423

13. The Unsteady Navier-Stokes Problem ............. 429

13.1 The Navier-Stokes Equations for Compressible and

Incompressible Flows $\ldots \ldots \ldots \ldots \ldots \ldots \ldots \ldots \ldots, 430$

13.1.1 Compressible Flows $\ldots \ldots \ldots \ldots \ldots \ldots \ldots \ldots \ldots, 431$

13.1.2 Incompressible Flows $\ldots \ldots \ldots \ldots \ldots \ldots \ldots \ldots, 432$ 
13.2 Mathematical Formulation and Behaviour of Solutions . .. 433

13.3 Semi-Discrete Approximation $\ldots \ldots \ldots \ldots \ldots \ldots \ldots \ldots, 434$

13.4 Time-Advancing by Finite Differences $\ldots \ldots \ldots \ldots \ldots \ldots .438$

13.5 Operator-Splitting Methods ................... 441

13.6 Other Approaches $\ldots \ldots \ldots \ldots \ldots \ldots \ldots \ldots \ldots \ldots \ldots, 446$

13.7 Complements $\ldots \ldots \ldots \ldots \ldots \ldots \ldots \ldots \ldots \ldots \ldots \ldots, 448$

14. Hyperbolic Problems $\ldots \ldots \ldots \ldots \ldots \ldots \ldots \ldots \ldots \ldots \ldots 449$

14.1 Some Instances of Hyperbolic Equations ............ 450

14.1.1 Linear Scalar Advection Equations ........... 450

14.1.2 Linear Hyperbolic Systems ............... 451

14.1.3 Initial-Boundary Value Problems $\ldots \ldots \ldots \ldots \ldots .453$

14.1.4 Nonlinear Scalar Equations . ............. 455

14.2 Approximation by Finite Differences $\ldots \ldots \ldots \ldots \ldots \ldots .461$

14.2.1 Linear Scalar Advection Equations and Hyperbolic Systems .......................... 461

14.2.2 Stability, Consistency, Convergence .......... 465

14.2.3 Nonlinear Scalar Equations $\ldots \ldots \ldots \ldots \ldots \ldots \ldots, 471$

14.2.4 High Order Shock Capturing Schemes ......... 475

14.3 Approximation by Finite Elements $\ldots \ldots \ldots \ldots \ldots \ldots \ldots .481$

14.3.1 Galerkin Method ..................... 482

14.3.2 Stabilization of the Galerkin Method ........... 485

14.3.3 Space-Discontinuous Galerkin Method .......... 487

14.3.4 Schemes for Time-Discretization ............. 488

14.4 Approximation by Spectral Methods ............. 490

14.4.1 Spectral Collocation Method: the Scalar Case ..... 491

14.4.2 Spectral Collocation Method: the Vector Case ..... 494

14.4.3 Time-Advancing and Smoothing Procedures ...... 496

14.5 Second Order Linear Hyperbolic Problems . . . . . . . . . . 497

14.6 The Finite Volume Method ...................... 501

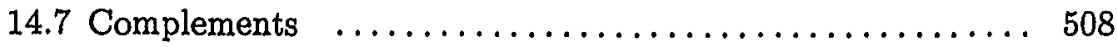

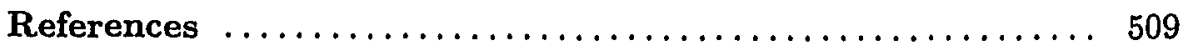

Subject Index $\ldots \ldots \ldots \ldots \ldots \ldots \ldots \ldots \ldots \ldots \ldots \ldots \ldots \ldots \ldots \ldots \ldots \ldots$ 